\title{
Welcome to Maple Transactions
}

\section{Recommended Reference Format:}

Laurent Bernardin. 2021. Welcome to Maple Transactions. Maple Trans. 1, 1, Article 14350 (2021), 1 page. https://doi.org/10.5206/mt.v1i1.14350

Maple was conceived over forty years ago as a general purpose system for mathematical calculations. Its strength, however, has always been its community. The work of hundreds of researchers from around the world has produced a mathematical engine unique in its depth, breath and efficiency. Forward thinking educators have used Maple to transform the way mathematics is taught, all the way supporting each other with advice, examples and myriads of Maple worksheets. Scientists and engineers have been taking advantage of the power and ease of use of the Maple system to help them in their discovery and the development of new products. Together we have tackled environmental issues, taken on disease and reached for the stars.

At Maplesoft, we are firm believers that Math Matters and our mission is to provide technology to explore, derive, capture, solve and disseminate mathematical problems and their applications, and to make math easier to learn, understand, and use. This mission, we share with hundreds of thousands of Maple users from all over the world and indeed we rely on that community's constant stream of feedback and support.

With Maple Transactions, our community is gaining a new place to come together. A place to exchange ideas, share experiences and discoveries. A place to welcome newcomers and discuss possibilities. The drive, vision and energy of editor in chief Prof Rob Corless together with the fantastic editorial board that he assembled, have given me a glimpse into a bright future for the journal and this first issue bears witness to the high quality of contributions we can expect.

Dr Laurent Bernardin

President \& CEO, Maplesoft 